\title{
Reverse Osmosis Technology, its Applications and Nano-Enabled Membrane
}

\author{
Ahamefula A. Ahuchaogu ${ }^{1}$, Okonkwo Joseph Chukwu' ${ }^{2}$, A. I. Obike ${ }^{1}$, Chitua E. Igara ${ }^{3}$, \\ Innocent Chidi Nnorom ${ }^{1}$, John Bull Onyekachi Echeme ${ }^{2}$ \\ ${ }^{1}$ Department of pure and industrial chemistry, Abia State University, Uturu Nigeria. \\ ${ }^{2}$ Department of chemistry, Michael Okpara University of Agriculture, Umudike, Umuahia, Abia State Nigeria, \\ ${ }^{3}$ Department of science Laboratory Technology, Akanu Ibiam Federal Polytechnic, Uwana Ebonyi State, \\ Nigeria
}

*Corresponding Author: Ahamefula A. Ahuchaogu, Department of pure and industrial chemistry, Abia State University, Uturu Nigeria.

\begin{abstract}
Reverse Osmosis (RO) is the finest of all membrane filtration system, it is a membrane based technology to purify water by separating the dissolved solids from feed stream resulting in permeate and reject stream for a wide range of applications in domestic as well as industrial applications. The objective of this paper is to review the concept of reverse osmosis and the current developments in the RO membrane materials which are the key determinants in the process. This review paper also presents the concept of membrane cleaning, emergence of nanomembranes and mixed matrix membrane materials as well as the various applications of $R O$ systems.
\end{abstract}

Keywords: Membrane materials, Osmosis, Reverse Osmosis (RO) Desalination, Nano-materials.

\section{INTRODUCTION}

Nowadays, desalination has become a very affordable solution to cope with fresh water shortage typically in tropical as well as off-shore areas. The desalination core process is based on Reverse Osmosis membrane technology.

Reverse Osmosis (RO) is a method of obtaining pure water from water containing a salt, as in desalination [1]. It is a water purification technology that uses a semi permeable membrane to remove ions, molecules and larger particles from drinking water. In reverse osmosis, an applied pressure is used to overcome osmotic pressure, a colligative property that is driven by chemical potential differences of the solvent, a thermodynamic parameter.

Reverse osmosis can remove many types of dissolved and suspended species from water, including bacteria and is used in both industrial processes and the production of potable water. The result is that the solute is retained on the pressurized side of the membrane and the pure solvent is allowed to pass to the other side. The membrane is expected to be selective not to allow large molecules or ions through the pores (holes), but should allow smaller components of the solution (such as solvent molecules) to pass freely [2].

In the normal osmosis process, the solvent naturally moves from the region of low solute concentration (high water potential), through a membrane, to a region of high solute concentration (low water potential). The driving force for the movement of the solvent is the reduction in the free energy of the system when the difference in solvent concentration on either side of a membrane is reduced, generating osmotic pressure due to the solvent moving into the more concentrated solution. Applying an external pressure to reverse the natural flow of pure solvent, thus, is reverse osmosis. Reverse osmosis also involves diffusion, making the process dependent on pressure, flow rate and other conditions [3]. It is most commonly known for its use in drinking water purification from sea water, removing the salt and other effluent materials from the water molecules. 
The process has been embraced by the world as a safe and affordable way to purify drinking water. It is effective in getting rid of dissolved chemicals and heavy metals such as: nitrate, sodium, crypto sporidium, fluoride, sulphides, giardia, arsenic, mercury, uranium, radium and lead. It is also effective in getting rid of harmful bacteria and chloride from water as it passes through the thin semi-permeable membranes. It is therefore important to review the recent developments in the area of reverse osmosis membranes.

\section{PROCESS DESCRIPTION}

When two solutions of different concentrations are separated by a semi-permeable membrane, the solvent (water) flows from the dilute solution to the concentrated solution till the concentrations of two solutions become equal. This process is known as osmosis.[4]

Formally, reverse osmosis is the process of forcing a solvent from a region of high solute concentration through a semi-permeable membrane to a region of low solute concentration by applying a pressure in excess of osmotic pressure. The largest and most important application of reverse osmosis is the separation of pure water from seawater and brackish water; seawater or brackish water is pressurized against one surface of the membrane, causing transport of salt - depleted water across the membrane and emergence of portable drinking water from the low pressure side.

The membranes used for reverse osmosis have a dense layer in the polymer matrix, either the skin of an asymmetric membrane or an interfacial polymerized layer within a thin-film composite membrane, where the separation occurs.

The RO process is simple in design consisting of feed, permeate and reject stream. For feed water, it is necessary to provide pretreatment in order to remove inorganic solids and suspended solids and using high pressure pump given feed through semi permeable membrane. Depending upon thepermeate where it is used, necessary post treatment is given[5]. A schematic diagram of the RO process is shown in figure 1.

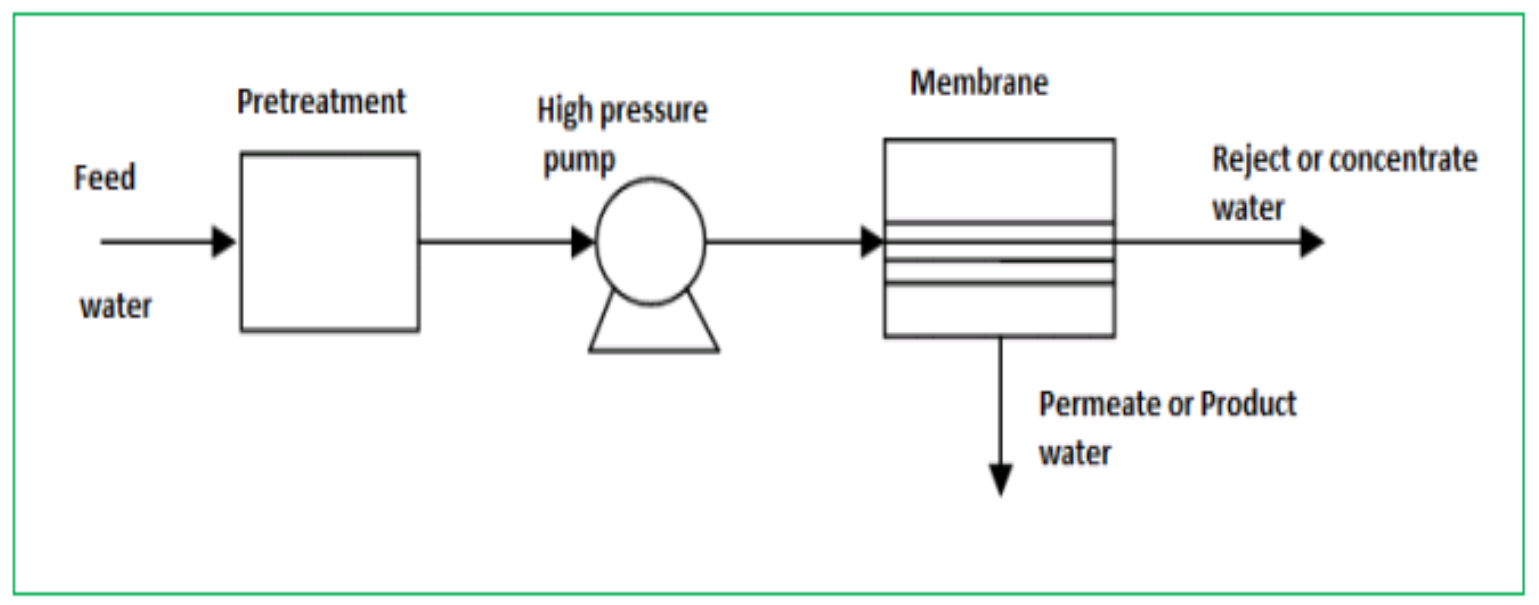

Figure1. Schematic Diagram of the RO process

\subsection{Reverse Osmosis Requisites}

Reverse osmosis desalination plant essentially consists of four major systems: (a) pretreatment system (b) high pressure pumps (c) membrane systems and (d) post-treatment. [5]. Pretreatment system is provided to remove all suspended solids so that salt precipitation or microbial growth does not occur on the membranes. Pretreatments may involve conventional methods like a chemical feed, followed by coagulation/flocculation/sedimentation and sand filtration or membrane processes i.e. micro filtration (MF) and ultra filtration (UF). High-pressure pumps supply the pressure needed to enable the water pass through the membrane and have the salt rejected. The pressure ranges from 17 to 27 bars for brackish water, and from 52 to 69 bars for seawater. Membrane systems consist of a pressure vessel and a semi permeable membrane inside that permits the feed water to pass through it. RO membranes for desalination generally come in two types: Spiral wound and hollow fiber. Depending upon water quality of permeate and use of permeate; post treatment may consist of adjusting the $\mathrm{pH}$ and disinfection [6]. 


\subsection{Advantages of RO Process}

Advantages of reverse osmosis that make it attractive for dilute aqueous wastewater treatment include:

(i) RO systems are simple to design and operate, have low maintenance requirements, and are modular in nature, making expansion of the systems easy.

(ii) Both organic and in organic pollutants can be removed simultaneously by reverse osmosis membrane processes.

(iii) RO systems require less energy as compared to other technology.

(iv) RO systems allow recovery/recycle of waste process streams with no effect on the material being recovered.

(v) The RO plant is normally operated at ambient temperature which reduces the scale formation and corrosion problems, because of antiscalent and biodispersent use, which will reduce maintenance cost.

(vi) The modular structure of the reverse osmosis process increases flexibility in building desalination plants within a wide range of capacities.

(vii) The specific energy requirement is significantly low $3-9.4 \mathrm{kw} / \mathrm{h} / \mathrm{m}^{3}$ product.

(viii) Reverse osmosis processes can considerably reduce the volume of waste streams so that there can be treated more efficiently and cost effectively by other processes such as incineration.

\subsection{Disadvantages}

i. Household reverse osmosis units use a lot of water because they have low back pressure. As a result, they recover only 5 to $15 \%$ of the water entering the system. The remainder is discharged as waste water. Because waste water carries with it the rejected contaminants, methods to recover this water are not practiced for household systems. Waste water is typically connected to the house drains and will add to the load on the household septic system. A reverse osmosis unit delivering five gallons (19L) of treated water per day may discharge between 20 and 90 gallons ( $75-340 \mathrm{~L}$ ) of waste water per day, [7] this may have disastrous consequence for mega cities.

ii. Due to its fine membrane construction, reverse osmosis not only removes harmful contaminants present in the water, but it also may remove many of the desirable minerals from the water. A number of peer-reviewed studies have looked at the long-term health effects of drinking demineralized water [8].

\subsection{Applications of Reverse Osmosis}

This technology has advantage of a membrane based process where concentration and separation is achieved without a change of state and without use of chemicals or thermal energy, thus making the process energy efficient and ideally suited for recovery applications. The bibliographic review shows applicability of reverse osmosis system for treating effluents from beverage industry, distillery spent wash, ground water treatment, and recovery of phenol compounds, drinking water purification, hydrogen production, window cleaning [9], reclamation of waste water and seawater reverse osmosis (SWRO) treatment indicating efficiency and applicability of RO technology [5].

\section{Membrane Technology}

Membranes are some of the most important parts of reverse osmosis. Most of the membranes used in commercial reverse osmosis plants are made from cellulose acetate, polyamide, polysulfonate and polyoxadiazole.

The membrane is usually made up of a 0.25 microns skin and 100 microns of support layer. The skin is usually the barrier that allows only water molecules to pass through. Murthy and Chaudhuri in 2009 reported on "Treatment of Distillery Spent washes" [10] where ultra-filtration and reverse osmosis membranes used for purification of the waste water by removing the colour and the contaminants. A number of studies such as Bellona et al., 2004 [11] and Xu et al., 2005 [12] have also reported on the application of reverse osmosis membranes for the removal of organics such as endocrine disrupting chemicals, plastic additives, pesticides, pharmaceutically active compounds, benzene and toluene. 
Cellulose acetate and polyamide membrane has good salt rejection for inorganic salts like $\mathrm{NaCl}$, $\mathrm{Na}_{2} \mathrm{SO}_{4}$. However, for organics, the rejection is reported and researched to be lower and varies widely in the range of $0.3-0.96[13,14]$.

\subsection{Conventional Desalination RO Membranes}

The conventional desalination RO membranes are polymeric materials such as polyamide spiral wound, asymmetric cellulose triacetate hollow fibre, aromatic polyamide-hydrazide, polypiperzineamide, polyoxadiazole etc. These polymeric RO membranes have dominated commercial applications since the very first RO desalination plant. Due to their technological maturity, they offer low-cost fabrication, ease of handling and improved performance in selectivity and permeability. One of the earliest review studies on polymeric RO membrane materials was reported by Cadotte in 1985[15]. It focused on composite RO membranes, covering activity from the inception of composite RO membranes up to approximately 1985. In 1993 Petersen [16]; offered a comprehensive review of the same subject, focusing on the chemistry of the membrane materials. Therefore for a complete study of early RO membrane development, readers are however advised to refer to Petersen 1993.

Generally, the development of membrane materials can be divided into two periods according to research activity:

i. The search for a suitable material (chemical composition, and membrane formation mechanism (1960s to late 1980s) and

ii. The evolution of more controlled conditions for membrane formulation to enhance membrane functionality and durability (late 1980s to date) [17].

\subsection{Membrane Characteristics}

The membrane should be in expensive, have longer and stable life. Membrane should be easily manufactured with good salt rejection, i.e. slightly permeable to salt. They should have high water flux, i.e. highly permeable to water and less susceptible to fouling. They should permit the flow of large amounts of water through the membrane relative to the volume they occupy. The membrane should be chemically, physically and thermally stable in saline waters, they need to be strong enough to withstand high pressures and variable feed water quality [5].

\subsection{Cleaning of a RO System Membrane}

The cleaning system is an important part of a desalination installation. Membranes can become contaminated after they have been used for some time, with pollutants such as colloids, bio films and biological matter. These contaminants can absorb to the membrane surface and the pipes of the membrane system and consequently, the performance of the system will decrease. The system may even be seriously damaged. That is why a system needs cleaning periodically.

Cleaning of a reverse osmosis is usually started when the following conditions are in order:

(a) The normalized flux has decrease $10-15 \%$

(b) The normalized salt content of the permeate has increased $10 \%$

(c) The pressure gradient in a pressure vessel decrease $15 \%$ [18].

The cleaning procedure of a reverse osmosis system consists of the following process steps.

i. Production of the cleaning fluid. The fluid that are used for the cleaning process need to be of a certain $\mathrm{pH}$ and all chemicals must be dissolved and mixed before the cleaning fluid is added in the membrane elements.

ii. The removal of feed water from pressure vessels and elements with cleaning fluid [18].

iii. Low flow recirculation through pressure vessels. The cleaning fluid is now in the drains and the feed water has been forced out of the system.

iv. Soaking in the cleaning fluid. The pump is shut off and the cleansing fluid will soak into the membranes. 
v. Drainage of the pressure vessels. The applied cleaning fluid is pumped out of the system. By sampling the cleansing fluid and analyzing the samples, one can determine the amount of contamination.

vi. Rinsing out the system. For the rinsing process, either permeates or good quality water is used.

vii. Starting up the cleaned system parts. The installation is started up according to the usual parameters. When cleaning fluid is still present in the permeate, the system needs to be rinsed again, until permeate quality is satisfactory [18].

\subsection{New Developments}

Since the 1970s, pre-filtration of high-fouling waters with another larger-pore membrane, with less hydraulic energy requirement has been evaluated and sometimes used. However, this means that the water passes through two membranes and is often re-pressurized which requires more energy to be put into the system, and thus increases the cost.

Other recent developmental work has focused on integrating reverse osmosis with electro dialysis to improve recovering of valuable deionized products, or to minimize the volume of concentrate requiring discharge or disposal.

In the production of drinking water, the latest developments include nanoscale and grapheme membranes [19].

\subsection{Nanomembranes}

Nanomembranes can be defined as filters that separate liquids and gases at the molecular level [20]. Nanotechnology which involves the development and use of devices that have a size of only a few nanometers. Research has been carried out into very small components, which depends on electronic effect and may involve movement of a countable number of electrons in their action. Such devices would act much faster than larger components. Considerable interest has been shown in the production of structures on a molecular level by suitable sequences of chemical reactions. It is also possible to manipulate individual atoms on surfaces using a variant of the atomic force microscope [1].

Although nanotechnology is a relatively new science, the idea of filtering at the nanoscale using a membrane is nothing new. It is a biomimetic process copied directly from nature. Living cells use a form of nanomembrane to function. These processes include the transfer of salts from blood and the transport of oxygen to and carbon dioxide from cells.

Nanomembranes are commonly made from organic polymer based nanocomposites with a thickness less than $100 \mathrm{~nm}$. Such nanomembranes include organic polymers combined with a mesh of silica nanoparticles. The size of the holes in the mesh restricts or allows the passage of different sized molecules [20].

\subsection{Mixed Matrix Membranes (MMM)}

The concept of mixed matrix membranes, the combination of organic and inorganic material, is also not new. Silicalite-cellulose acetate MMM for gas separation was developed in 1980, showing superior selectivity to conventional polymeric membranes [17]. Despite the fact that MMM were developed for water/ethanol separation via pervaporation in the 1990s, the incorporation of inorganic materials into organic reverse osmosis - thin film composite membranes only started in the early 2000s.

The main objective of MMM is to combine the benefits offered by each material, i.e. the high packing density, good permselectivity and long operational experience of polymeric membranes, coupled with the superior chemical, biological and thermal stability of inorganic membranes. Examples of such membranes are nano-particle/polymeric membranes, $\left(\mathrm{Ti}_{2} \mathrm{O}_{2}\right.$ nano particle/polymeric membrane, zeolite nanoparticle/polymeric membrane) and carbon nanotube/polymeric membrane (CNT) [17].

\subsection{Fabricating Nanomembranes}

Nanomembranes are commonly fabricated using layer-by-layer (LBL) assembly methods. This method give precise control over the in plane composition of the membrane and allows for the 
addition of a range of components to be added to the membrane. These components include nanoparticles and nanotubes that can tailor the mechanical, optical and electronic properties of the nanomembrane [20].

\section{CONCLUSION}

Reverse osmosis process has been embraced by the world as a safe and affordable way to purify drinking water, since the process is effective in getting rid of dissolved chemicals, heavy metals, getting rid of harmful bacteria and chlorine from water as they pass through the thin semi-permeable membranes. RO is also energy efficient as it doesn't require use of electricity, all it requires is sufficient water pressure to power it through the entire process. Therefore, priority should be given to the membrane maintenance and cleaning as well as move extensive search for multi-functional membrane materials that offer higher permeability, high ion and organic contaminants rejection.

\subsection{Future Scope}

Currently, the two practical challenges limiting the advance in the nano-structured membrane materials are the high cost of nanomaterials and the difficulty in scaling up nano-membrane manufacturing processes for commercial use. In addition, health and safety issues around the use of nano-materials. Therefore the key future area of study in RO desalination technology should target at development single-pass reverse osmosis using multifunctional membranes, eliminating the need for pretreatment, development of novel RO-membranes with improved salt rejection and permeability at a reasonable maintenance, capital investment and operating cost.

\section{REFERENCES}

[1] David, E. W.; Richard, R.; Edwards, D. E.; Derek, C.; Cutter, R.; Clarke, M. H.; and John, C. (2004). Oxford dictionary of chemistry Oxford university press, New York $5^{\text {th }}$ ed. Pp. 486.

[2] Warsinger, david M.; Tow, Emily, W.; Nayar, Kishor G.; Maswadeh, Laith A.; Lienhard V, John H. (2016). "Energy efficiency of batch and semi-batch (CCRO) reverse osmosis desalination". Water research pp. $272-282$.

[3] Crittenden, john;Trussell, Rhodes; Hand, David; Howe, Kerry and Tchobanoglous, George (2005). Water treatment principles and designs, $2^{\text {nd }}$ ed. John Wiley and Sons. New Jersey. ISBN 0-471-11018-3.

[4] S.C. Bhatia (2008). Engineering Chemistry. $1^{\text {st }}$ ed. CBS publisher and distributors. New Delhi (India). Pp.49.

[5] Garud, R.M., Kore, S.V., Kore, V.S., and Kulkarni G.S. (2011): "A short review on process and application of reverse osmosis" universal journal of environmental research and technology, vol. 1 3:233 238.

[6] Djebedjian, B., Gad, H.E., Khaled, I., and About Rayan, M., "An Experimental investigation on Reverse Osmosis Desalination system, " Engineering conference, Sharam El-Sheikh, Egypt, 27-31 march,2006,pp. M-99-111.

[7] Treatment systems for household water supplies.ag.ndsu.ed retrieved on 2011-06-19.

[8] Health risk from drinking demineralized water.

[9] "Government versus Gravity" retrieved 2013-06-24.

[10] Murthy, G. V.P and Choudhuri, L.B, (2009). Treatment of distillery spent wash by combined UF and RO processes, global NEST journal, vol. 11, 2:235 - 240.

[11] Bellona, C., J.E. Drewes, P. Xua and G. Amy (2004). Factors affecting the rejection of organic solutes during NF/Ro treatment - a literature review, water research, 38:2795 - 2809.

[12] Xu, P., J. E. Drewes, C. Bellona, G. amy and T. U. Kim et al (2005), rejection of emerging organic micro pollutants in nanofiltration reverse osmosis membrane application water environment research, 77:40 - 48.

[13] Pozderivic, A. T. Moslavac and A. Pichler (2006), concentration fo aqueous solution of organic components by reverse osmosis II. Influence of tranmembrane pressure and membrane type on concentration of different alcohol solutions by reverse osmosis, journal of food engineering, 77:810 - 81.

[14] Senthilmurugan, S. and S. K. Gupta (2006), separation of inorganic and organic compounds by using a radial flow hollow fiber reverse module, desalination, 196:221 - 236 .

[15] J. E. Cadotte (1985). Evolution of composite reverse osmosis membranes: materials science of synthetic membrane. Based on a symposium at the $187^{\text {th }}$ meeting of the American chemical society, Acs, St Louis, MO, USA, pp.273-294. 
[16] Petersen, R.J (1993) composite reverse osmosis and nanofiltration membranes, journal of membrane sciences, 83. Pp.81 - 150 .

[17] Keh Peng, L. Tom Arnot C. Davide, M. (2010) “A review of reserve osmosis membrane materials for desalination - development to date and future potential" journal of membrane science, 370 (1-2): 1 - 22.

[18] www.lenntech.com/cleaning-or-systems

[19] Zhu, Chongqin, Li. Hui, Zeng, Xiao Chng, wang, E.G.; Meng, Sheng (2013). Quantized water transport ideal desalination through Graphyne - 4 membrane" scientific report, 3:3163.

[20] www.azonano/article.aspx?article ID=1834

Citation: A. Ahuchaogu et al., "Reverse Osmosis Technology, its Applications and Nano-Enabled Membrane", International Journal of Advanced Research in Chemical Science (IJARCS), vol. 5, no. 2, pp. 2026, 2018. http://dx.doi.org/10.20431/2349-0403.0502005

Copyright: (C) 2018 Authors. This is an open-access article distributed under the terms of the Creative Commons Attribution License, which permits unrestricted use, distribution, and reproduction in any medium, provided the original author and source are credited. 